\title{
Coronavirus disease 2019: initial chest CT findings
}

\section{Zhiming Zhou ${ }^{1} \cdot$ Dajing Guo ${ }^{1} \cdot$ Chuanming $\mathrm{Li}^{1} \cdot$ Zheng Fang $^{1} \cdot$ Linli Chen ${ }^{1} \cdot$ Ran Yang $^{2} \cdot$ Xiang $\mathrm{Li}^{2} \cdot$ Wenbing Zeng $^{2}$}

Received: 13 February 2020 / Revised: 12 March 2020 / Accepted: 16 March 2020 / Published online: 24 March 2020

(C) European Society of Radiology 2020

\begin{abstract}
Objectives To systematically analyze CT findings during the early and progressive stages of natural course of coronavirus disease 2019 and also to explore possible changes in pulmonary parenchymal abnormalities during these two stages.

Methods We retrospectively reviewed the initial chest CT data of 62 confirmed coronavirus disease 2019 patients ( 34 men, 28 women; age range 20-91 years old) who did not receive any antiviral treatment between January 21 and February 4, 2020, in Chongqing, China. Patients were assigned to the early-stage group (onset of symptoms within 4 days) or progressive-stage group (onset of symptoms within 4-7 days) for analysis. CT characteristics and the distribution, size, and CT score of pulmonary parenchymal abnormalities were assessed.

Results In our study, the major characteristic of coronavirus disease 2019 was ground-glass opacity (61.3\%), followed by ground-glass opacity with consolidation (35.5\%), rounded opacities $(25.8 \%)$, a crazy-paving pattern (25.8\%), and an air bronchogram $(22.6 \%)$. No patient presented cavitation, a reticular pattern, or bronchial wall thickening. The CT scores of the progressive-stage group were significantly greater than those of the early-stage group $(p=0.004)$.

Conclusions Multiple ground-glass opacities with consolidations in the periphery of the lungs were the primary CT characteristic of coronavirus disease 2019. CT score can be used to evaluate the severity of the disease. If these typical alterations are found, then the differential diagnosis of coronavirus disease 2019 must be considered.

Key Points

- Multiple GGOs with consolidations in the periphery of the lungs were the primary CT characteristic of COVID-19.

- The halo sign may be a special CT feature in the early-stage COVID-19 patients.

- Significantly increased CT score may indicate the aggravation of COVID-19 in the progressive stage.
\end{abstract}

Keywords COVID-19 $\cdot$ SARS-CoV-2 $\cdot$ Coronavirus $\cdot$ Pneumonia $\cdot$ Spiral CT scan

Abbreviations
COVID-19
CRP
GGO
MERS-CoV

Coronavirus disease 2019

C-reactive protein

Ground-glass opacity

MERS-CoV Middle East respiratory

syndrome coronavirus

MPR Multiplanar reconstruction

RT-PCR Reverse transcription polymerase

chain reaction

Wenbing Zeng

zeng_wenbing@163.com

1 Department of Radiology, the Second Affiliated Hospital of Chongqing Medical University, Chongqing, China

2 Department of Radiology, Chongqing Three Gorges Central Hospital, Chongqing 404000, China

$\begin{array}{ll}\text { SARS } & \begin{array}{l}\text { Severe acute respiratory syndrome } \\ \text { Severe acute } \\ \text { respiratory syndrome coronavirus }\end{array} \\ \text { SARS-CoV-2 } & \begin{array}{l}\text { Severe acute respiratory syndrome } \\ \text { coronavirus 2 }\end{array} \\ \text { WHO } & \text { World Health Organization }\end{array}$

\section{Introduction}

Since December 2019, an increasing number of pneumonia cases caused by the severe acute respiratory syndrome coronavirus 2 (SARS-CoV-2) emerged in Wuhan, Hubei, China, and subsequently, an outbreak of coronavirus disease 2019 (COVID-19) swept the globe [1-4]. As of February 3, 2020, more than 23,214 suspected cases and 20,438 confirmed cases have been identified, and there has been over 425 deaths 
related to the illness. Moreover, the virus is still spreading at an alarming rate.

In this sudden epidemic, reverse transcription polymerase chain reaction (RT-PCR) revealed the cause of the outbreak. The SARS-CoV-2 can be specifically detected in respiratory secretions or plasma samples RT-PCR. However, some patients with likely COVID-19 may have negative initial RTPCR results for COVID-19, which may be due to insufficient cellular material for detection or improper extraction of nucleic acids from clinical materials. Thus, imaging examination, which can effectively and conveniently observe the pulmonary parenchymal abnormalities, can solve some challenges associated with clinical diagnosis in suspected patients with a false-negative result, especially symptomatic patients with dyspnea and respiratory distress.

Because this infection is caused by the unprecedented SARS-CoV-2, few related imaging studies have been currently reported. Among the studies that have been conducted, multifocal consolidation or ground-glass opacity (GGO) was observed on chest radiographs and CT [5-7]. However, the sensitivity of chest radiographs for pneumonia are relatively low, and it is difficult to use chest radiographs to distinguish COVID-19 from common pneumonia during the cold season and pneumonia caused by other coronaviruses, such as severe acute respiratory syndrome coronavirus (SARS-CoV) and Middle East respiratory syndrome coronavirus (MERS-CoV) infections [8]. In addition, the disease is developing rapidly and the symptoms of this disease vary. Hence, it is very necessary to systematically analyze the chest CT findings associated with this disease systematically, for the timely isolation, COVID-19 RT-PCR and respiratory care of patients, and early implementation of infection prevention and control measures.

To fully understand and early discriminate the $\mathrm{CT}$ features of this disease in its early stages, we collected initial chest CT data from confirmed COVID-19 patients who did not receive any antiviral treatment mainly from Chongqing Three Gorges Central Hospital for analysis. The Chongqing Three Gorges Central Hospital, located in the northeast of Chongqing and connected to Hubei Province, is one of the designated hospitals for the centralized treatment of pneumonia patients infected by the SARS-CoV-2 and has received a large number of COVID19 patients. Therefore, our aim was to systematically analyze the CT features of the early and progressive stages of the natural course of COVID-19 and explore the possible pathological changes in the lung during different periods of this disease.

\section{Materials and methods}

\section{Study participants}

This retrospective study was approved by the Institutional Ethics Committee of Chongqing Three Gorges Central
Hospital and the Second Affiliated Hospital of Chongqing Medical University, and the requirement for informed consent was waived.

Between January 21 and February 4, 2020, totally 72 patients of confirmed COVID-19 were enrolled, i.e., 66 patients from Chongqing Three Gorges Central Hospital and 6 patients from the Second Affiliated Hospital of Chongqing Medical University. All patients with COVID-19 were diagnosed according to the World Health Organization (WHO) interim guidance [9] and were eventually confirmed by a positive result for RT-PCR analysis of the respiratory secretions samples. Confirmed patients were eligible if they experienced symptoms within 7 days, underwent an initial chest CT examination, and they had not received any antiviral treatment. The exclusion criteria were as follows: (1) patients who recently experienced clinically defined pulmonary infection attributable to other pathogens, (2) patients with severe artifacts on $\mathrm{CT}$ images, and (3) patients whose age was less than 18 years. According to the exclusion criteria, 7 patients who had pulmonary infections caused by other pathogens and 3 patients whose $\mathrm{CT}$ images had severe artifacts were excluded. Finally, 62 confirmed COVID-19 patients (34 men, 28 women; age range 20-91 years old) who had not received antiviral treatment were retrospectively reviewed. The workflow of COVID-19 patients selection is shown in Fig. 1. Most cases had a history of SARS-CoV-2 exposure, in the absence of medical staff or family clusters of SARS-CoV-2 infection. The main symptom of patients was fever, and secondary symptoms included cough, expectoration, dizziness, nasal congestion, and myalgia. According to the changes in this disease over time [10,11], in our study, we defined patients with an onset of symptoms within 4 days as the early-stage group (18 men, 16 women; age range 20 72 years old) and patients with an onset of symptoms within 5-7 days as the progressive-stage group (16 men, 12 women; age range 22-91 years old). All patients underwent CT scans and laboratory examinations for C-reactive protein (CRP), white blood cell count, neutrophil count, lymphocyte count, platelet count, hemoglobin level, and procalcitonin level on admission.

\section{CT data acquisition}

Designated independent operating rooms were used to perform the CT examinations of all confirmed patients and after each examination; the operating room was sterilized with air disinfection method. The initial chest CT data of 56 patients from Chongqing Three Gorges Central Hospital were obtained by using a 16-slice spiral CT scanner (Emotion 16 VC20B, Siemens Healthcare $\mathrm{GmbH}$ ) with the following scanning protocol: a tube voltage of $120 \mathrm{kV}$, smart $\mathrm{mA}$ tube current modulation, a slice thickness of $1.5 \mathrm{~mm}$, and a detector width of $1.5 \mathrm{~mm}$. The initial chest 
Fig. 1 The workflow of coronavirus disease 2019 selection

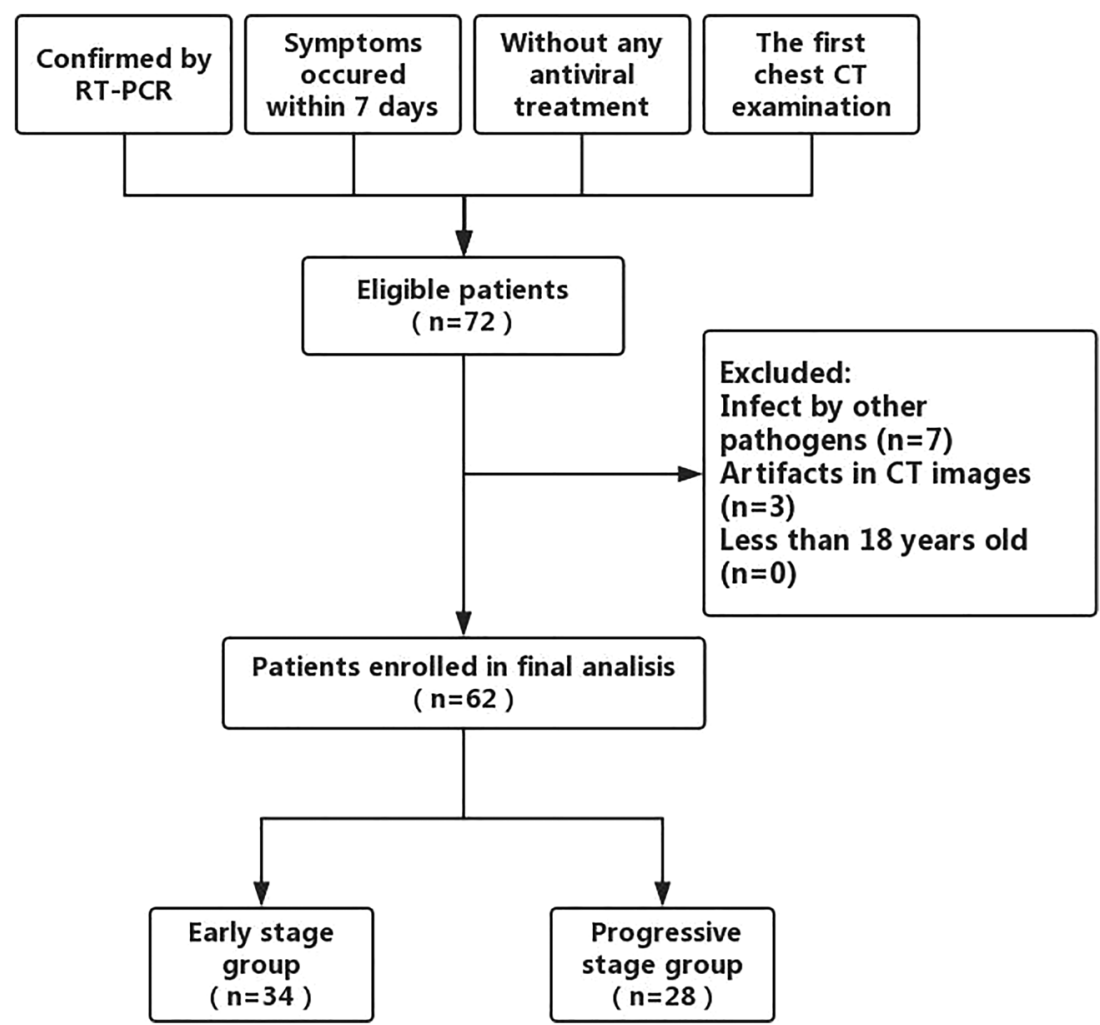

CT data of the other 6 patients from the Second Affiliated Hospital of Chongqing Medical University were obtained by using a 128-slice spiral CT scanner (Ingenuity CT, Philips Healthcare) with a similar scanning protocol: a tube voltage of $120 \mathrm{kV}$, smart $\mathrm{mA}$ tube current modulation, a matrix size of $512 \times 512$, a slice thickness of $1 \mathrm{~mm}$, and a detector width of $0.625 \mathrm{~mm}$. All patients were scanned in the supine position and at the suspended end-inspiratory volume, and the entire lung was scanned from the apex to the bottom [12]. To reduce discrepancies in the image acquisition parameters attributable to the different CT scanners, we resampled all voxels at $1.0 \times 1.0 \times 1.0 \mathrm{~mm}^{3}$ before image analysis. The multiplanar reconstruction (MPR) technique was performed to better observe images at the postprocessing station when necessary.

\section{CT data analysis}

All CT findings were described according to the Fleischner Society recommendations and defined as follows: GGO (appears as hazy increased opacity in the lung, with the preservation of bronchial and vascular margins), consolidation (appears as a homogeneous increase in pulmonary parenchymal attenuation that obscures the margins of vessels and airway walls), a crazy-paving pattern (appears as thickened interlobular septa and intralobular lines superimposed on a background of GGO), and a halo sign (appears as GGO surrounding a nodule or mass) $[13,14]$. Attention was paid to CT images with GGO, consolidation, GGO with consolidation, a reticular pattern, nodules, rounded opacities, cavitation, a crazy-paving pattern, an air bronchogram, a halo sign, bronchial wall thickening, a subpleural curvilinear line, and pulmonary fibrosis, and the presence, distribution, and size of these CT characteristics were assessed in all subjects. In addition, the presence of thoracic lymphadenopathy (lymph node size of greater than or equal to $10 \mathrm{~mm}$ in the short-axis dimension) and pleural effusion or thickening were also recorded. The distribution of each finding was classified as follows: central (predominantly in the inner two-thirds of the lung), peripheral (predominantly in the outer third of the lung), and diffuse (indications in multiple lung segments) [15]. Calculation of the CT score, a semiquantitative evaluation method, was applied to quantify the extent of COVID-19 according to previous studies $[15,16]$. First, the scope of the lesions in each lobe was estimated and a score of 0 (none), 1 (affecting less than $5 \%$ of the lobe), 2 (affecting 5-25\% of the lobe), 3 (affecting 26-49\% of the lobe), 4 (affecting 50-75\% of the lobe), or 5 (affecting more than $75 \%$ of the lobe) was assigned. Second, the CT score was obtained by adding up the scores of the five lobes. For each patient, the CT score was in the range of 0 to 25 . All $\mathrm{CT}$ scans were independently reviewed and assessed by two chest radiologists (Z.F. and L.L.C., who had 18 years of experience in thoracic radiology, respectively). Disagreements in reader interpretation were settled by consensus agreement. 


\section{Statistical analysis}

All of the data were analyzed with statistical software (SPSS statistical package, version 19.0; IBM Corporation). Continuous variables were expressed as means \pm standard deviations or median (interquartile range (IQR)), and categorical variables were presented as numbers (percentages). Kolmogorov-Smirnov tests were applied to check the normality of variables. Mann-Whitney $U$ tests were used for nonnormally distributed data. Two-sample $t$ tests were used for normally distributed data. Chi-square tests or Fisher's exact tests were applied to compare categorical variables. Spearman's correlation was performed to analyze the relationship between clinical variables (age, fever, CRP, white blood cell count, neutrophil count, lymphocyte count, platelet count, hemoglobin level, and procalcitonin level) and CT variables (maximum diameter of the lesion and CT score). For all test results, a $p$ value of less than 0.05 was considered statistically significant.

\section{Results}

\section{Demographic and clinical characteristics of patients with COVID-19}

The demographic and clinical characteristics of all patients with COVID-19 are summarized in Table 1. Among all 62 patients, $51(82.3 \%)$ patients had a confirmed history of SARS-CoV-2 exposure, 43 (69.4\%) patients had fever, and mainly low-grade fever $(46.8 \%)$. There were no significant differences in terms of age, sex, and laboratory test results (CRP, white blood cell count, neutrophil count, lymphocyte count, platelet count, hemoglobin level, procalcitonin level) between the early-stage group and progressive-stage group (all $p>0.05$ ).

\section{CT findings in patients with COVID-19}

The CT findings of patients with COVID-19 in early and progressive stages are listed in Table 2 and shown in Figs. 2, 3,4 , and 5 . In terms of CT characteristics, the major finding was the GGO in $38(61.3 \%)$ of the 62 patients. In addition, GGO with consolidation was observed in 22 (35.5\%) patients; rounded opacities were observed in $16(25.8 \%)$ patients; a crazy-paving pattern was observed in $16(25.8 \%)$ patients; an air bronchogram was observed in $14(22.6 \%)$ patients; a halo sign was observed in 7 (11.3\%) patients; a subpleural curvilinear line was observed in $6(9.7 \%)$ patients; pleural effusion was observed in $2(3.2 \%)$ patients; and consolidation, nodules, thoracic lymphadenopathy, and pulmonary fibrosis were observed in $1(1.6 \%)$ patient each. No patient presented cavitation, a reticular pattern, or bronchial wall thickening. Between the early-stage group and progress-stage group, there were no significant differences in the CT findings, except for the halo sign ( $p=0.013$ ). Specifically, the halo sign was found in only the early-stage group in $20.6 \%$ of the patients.

In terms of lesion size, lesions with a maximum diameter greater than $5 \mathrm{~cm}$ were found in $21(33.9 \%)$ of 62 patients; lesions with a maximum diameter of $3-4.9 \mathrm{~cm}$ were found in $22(35.5 \%)$ patients; lesions with a maximum diameter of $1-$ $2.9 \mathrm{~cm}$ were found in 18 (29.0\%) patients; and lesions with a maximum diameter less than $1 \mathrm{~cm}$ were found in $1(1.6 \%)$

Table 1 Demographic and clinical characteristics between two groups in patients with COVID-19

\begin{tabular}{|c|c|c|c|}
\hline Variables & Early-stage group $(n=34)$ & Progressive-stage group $(n=28)$ & $p$ value \\
\hline \multicolumn{4}{|l|}{ Demographic } \\
\hline Age, (years) & $44.32 \pm 13.53$ & $50.82 \pm 13.23$ & 0.062 \\
\hline Male & $18(52.9 \%)$ & $16(57.1 \%)$ & $0.741(\chi)$ \\
\hline Exposure history & & & $0.494(\chi)$ \\
\hline Positive & $27(79.4 \%)$ & $24(85.7 \%)$ & \\
\hline Negative & $4(11.8 \%)$ & $1(3.6 \%)$ & \\
\hline Unknown & $3(8.8 \%)$ & $3(10.7 \%)$ & \\
\hline \multicolumn{4}{|l|}{ Symptoms } \\
\hline Fever $\left({ }^{\circ} \mathrm{C}\right)$ & $37.70 \pm 0.73$ & $37.66 \pm 0.94$ & 0.854 \\
\hline$<37.4{ }^{\circ} \mathrm{C}$ & $8(23.5 \%)$ & $11(39.3 \%)$ & $0.064(\chi)$ \\
\hline $37.4-38^{\circ} \mathrm{C}$ & $20(58.8 \%)$ & $9(32.1 \%)$ & \\
\hline $38.1-39^{\circ} \mathrm{C}$ & $4(11.8 \%)$ & $6(21.4 \%)$ & \\
\hline $39.1-41^{\circ} \mathrm{C}$ & $2(5.9 \%)$ & $2(7.1 \%)$ & \\
\hline \multicolumn{4}{|l|}{ Laboratory tests } \\
\hline $\mathrm{CRP}(\mathrm{mg} / \mathrm{L})$ & $15.98(1.78-55.98)$ & $35.62(5.63-63.04)$ & 0.329 \\
\hline White blood cell count $\left(\times 10^{9} / \mathrm{L}\right)$ & $5.78 \pm 1.93$ & $6.24 \pm 3.55$ & 0.518 \\
\hline Neutrophil count $\left(\times 10^{9} / \mathrm{L}\right)$ & $0.71 \pm 0.13$ & $0.74 \pm 0.11$ & 0.343 \\
\hline Lymphocyte count $\left(\times 10^{9} / \mathrm{L}\right)$ & $1.04 \pm 0.49$ & $0.97 \pm 0.46$ & 0.541 \\
\hline Platelet count $\left(\times 10^{9} / \mathrm{L}\right)$ & $180.79 \pm 66.83$ & $190.18 \pm 73.91$ & 0.602 \\
\hline Hemoglobin $(g / d L)$ & $13.72 \pm 1.79$ & $13.69 \pm 1.80$ & 0.956 \\
\hline Procalcitonin (ng/mL) & $0.05(0.04-0.07)$ & $0.07(0.04-0.11)$ & 0.234 \\
\hline
\end{tabular}

COVID-19, coronavirus disease 2019; CRP, C-reactive protein 
Table 2 Comparison of CT findings and scores between two groups in patients with COVID-19

\begin{tabular}{|c|c|c|c|}
\hline Variables & Early-stage group $(n=34)$ & Progressive-stage group $(n=28)$ & $p$ value \\
\hline \multicolumn{4}{|l|}{ CT findings } \\
\hline Ground-glass opacity & $22(64.7 \%)$ & $16(57.1 \%)$ & $0.543(\chi)$ \\
\hline Consolidation & $1(2.9 \%)$ & $0(0 \%)$ & $1.000(\chi)$ \\
\hline Ground-glass opacity with consolidation & $11(32.4 \%)$ & $11(39.3 \%)$ & $0.570(\chi)$ \\
\hline Reticular pattern & 0 & 0 & - \\
\hline Nodule & $1(2.9 \%)$ & 0 & $1.000(\chi)$ \\
\hline Rounded opacities & $10(29.4 \%)$ & $6(21.4 \%)$ & $0.475(\chi)$ \\
\hline Cavitation & 0 & 0 & - \\
\hline Crazy-paving pattern & $8(23.5 \%)$ & $8(28.6 \%)$ & $0.652(\chi)$ \\
\hline Air bronchogram & $8(23.5 \%)$ & $6(21.4 \%)$ & $0.844(\chi)$ \\
\hline Halo sign & $7(20.6 \%)$ & 0 & $0.013(\chi)$ \\
\hline Bronchial wall thickening & 0 & 0 & - \\
\hline Subpleural curvilinear line & $4(11.8 \%)$ & $2(7.1 \%)$ & $0.681(\chi)$ \\
\hline Thoracic lymphadenopathy & 0 & $1(3.6 \%)$ & $0.452(\chi)$ \\
\hline Pleural effusion or thickening & $1(2.9 \%)$ & $1(3.6 \%)$ & $1.000(\chi)$ \\
\hline Pulmonary fibrosis & 0 & $1(3.6 \%)$ & $0.452(\chi)$ \\
\hline Max diameter of lesion $(\mathrm{cm})$ & $4.27 \pm 2.66$ & $5.83 \pm 3.76$ & 0.059 \\
\hline$<1 \mathrm{~cm}$ & 0 & $1(3.6)$ & $0.201(\chi)$ \\
\hline $1-2.9 \mathrm{~cm}$ & $13(38.2 \%)$ & $5(17.9 \%)$ & \\
\hline $3-4.9 \mathrm{~cm}$ & $12(35.3 \%)$ & $10(35.7 \%)$ & \\
\hline$>5 \mathrm{~cm}$ & $9(26.5 \%)$ & $12(42.9 \%)$ & \\
\hline \multicolumn{4}{|l|}{ Distribution } \\
\hline Central & $1(2.9 \%)$ & $1(3.6 \%)$ & $0.961(\chi)$ \\
\hline Peripheral & $12(35.3 \%)$ & $9(32.1 \%)$ & \\
\hline Both central and peripheral & $21(61.8 \%)$ & $18(64.3 \%)$ & \\
\hline CT score & $4.79 \pm 2.76$ & $7.79 \pm 4.62$ & 0.004 \\
\hline $0-5$ & $27(79.4 \%)$ & $10(35.7 \%)$ & $0.004(\chi)$ \\
\hline $6-10$ & $6(17.6 \%)$ & $12(42.9 \%)$ & \\
\hline $11-15$ & 0 & $3(10.7 \%)$ & \\
\hline$>15$ & $1(2.9 \%)$ & $3(10.7 \%)$ & \\
\hline
\end{tabular}

COVID-19, coronavirus disease 2019

patient. There was no statistically significant difference in lesion size between the two groups of patients with COVID-19 ( $p=0.059)$. Regarding the distribution of pulmonary parenchymal abnormalities, lesions that were mainly distributed in both the central and peripheral lung regions accounted for $62.9 \%$; lesions distributed in the peripheral lung were present in $21(33.9 \%)$ patients; and lesions distributed in the central lung were present in $2(3.2 \%)$ patients. There was no statistically significant difference in the distribution of pulmonary parenchymal abnormalities between the two groups of patients with COVID-19 $(p=0.961)$.

\section{CT score of patients with COVID-19}

The CT scores of all patients with COVID-19 are shown in Table 2 and the CT scores of each lobe in the two groups are shown in Fig. 6. The CT scores of the progressive-stage group were significantly greater than those of the earlystage group $(p=0.004)$. In the early-stage group, 27 (79.4\%) patients had CT scores in the range of $0-5,6$ (17.6\%) patients had CT scores of 6-10, and only 1 patient had a CT score of 11 or more. In the progressive-stage group, $10(35.7 \%)$ patients had CT scores in the range of $0-5,12$ (42.9\%) patients had CT scores of 6-10, 3 (10.7\%) patients had CT scores of 11-15, and $3(10.7 \%)$ patients had CT scores of 15 or more.

Among all 62 COVID-19 patients, the CT scores of pulmonary parenchymal abnormalities were significantly positively correlated with maximum diameter of the lesions $(r=$ $0.531, p<0.001$ ), and the CT scores were also significantly positively correlated with the age of the patients $(r=0.255$, $p=0.045$ ), shown in Table 3 . 
Fig. 2 A 56-year-old male with coronavirus disease 2019 in the early-stage group. a Axial noncontrast CT shows ground-glass opacities in the anterior segments of bilateral upper lobes. b Ground-glass opacity is also shown in the peripheral region of the lateral basal segment of left lower lobe. $\mathrm{CT}$ score $=1$ (right upper lobe) +1 (left upper lobe $)+1$ (left lower lobe $)=3$
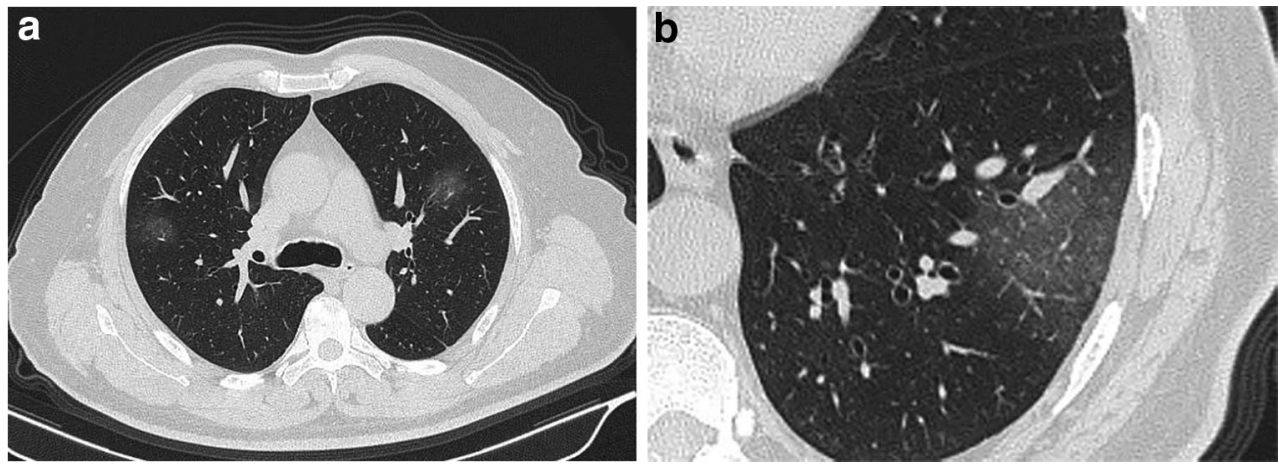

\section{Discussion}

As reported, patients with COVID-19 usually develop clinical symptoms after an incubation period of 4.1-7.0 days [11]. After admission, chest imaging examination was performed on them. According to the results of changes in this disease in recent studies $[10,11]$, the patient's condition progressively worsens on the $3-5$ days after symptom onset, and the guidelines for an imaging diagnosis of COVID-19 recommend that reviewing CT or plain radiograph is needed for suspected cases 35 days after diagnosis to observe changes in the disease status [12]. Thus, we assigned patients to two groups according to the time of onset of symptoms to analyze the CT findings in earlystage and progressive-stage of COVID-19. In this study, 62 confirmed patients with COVID-19 underwent chest CT scans, and positive rate of detecting the pulmonary parenchymal abnormalities was $100 \%$. In addition, the CT data in our study were obtained from the initial chest CT examination of patients who had not received any antiviral therapy; these results may better reflect the degree of viral infection and the immune system status before clinical intervention and provide an important basis for clinical classification, treatment strategy development, and prognosis prediction.

In our study, the major characteristic of COVID-19 was GGO (61.3\%), followed by GGO with consolidation (35.5\%), rounded opacity (25.8\%), a crazy-paving pattern

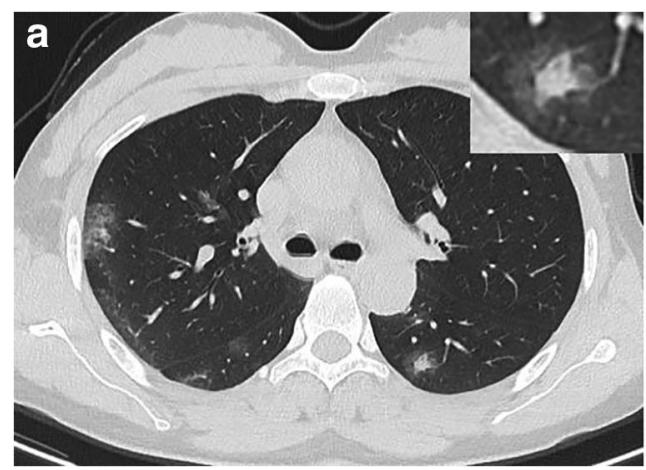

Fig. 3 A 34-year-old female with coronavirus disease 2019 in the earlystage group. a Axial non-contrast CT shows halo sign in the dorsal segment of left lower lobe and multiple ground-glass opacities in the right upper lobe and the dorsal segment of the right lower lobe. b Sagittal non-
(25.8\%), and an air bronchogram (22.6\%). In contrast, pulmonary fibrosis $(1.6 \%)$ and pleural effusions (3.2\%) were rare on chest $\mathrm{CT}$ images. These various $\mathrm{CT}$ findings are likely related to the complex pathological changes in the lungs of patients with COVID-19. Although pathological changes in the lung tissue of COVID-19 patients have not yet been studied, recent studies have reported that SARS-CoV-2 is closely related (with $88 \%$ identity) to two bat-derived severe acute respiratory syndrome (SARS)-like coronaviruses, with approximately $79 \%$ homology with SARS-CoV and approximately 50\% homology with MERS-CoV $[17,18]$. Because the pathogenesis of viral infections in the same family may be similar, previous studies on coronaviruses may be helpful in understanding the various CT findings in COVID-19 patients based on similar pathological changes. In SARS patients, pathological changes include injury to pulmonary epithelial cells, hyaline membrane formation on in the inner wall of the alveoli, and a large number of tissue cells and mass thrombi formed by proliferative fibrous tissue that block the small airway and air cavity [19]. These pathological changes may be the main pathological basis of the CT findings, especially the GGO.

Among all the CT findings, GGO was the most common one in both the early and progressive stages of COVID-19 and was characterized by multiple sized lesions, mainly distributed in the peripheral lung (96.8\%). An analysis of thin-section CT images of SARS patients showed that multiple GGOs

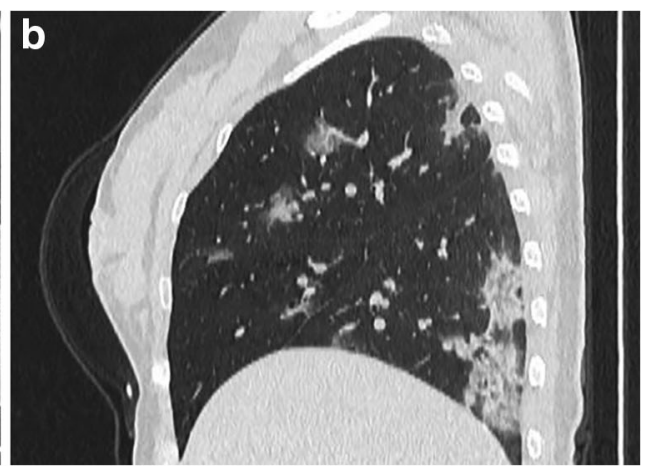

contrast CT demonstrates multiple ground-glass opacities with consolidations in the right lung, mainly distributed in the periphery. CT score $=2$ $($ right upper lobe $)+2($ right lower lobe $)+1($ left lower lobe $)=5$ 


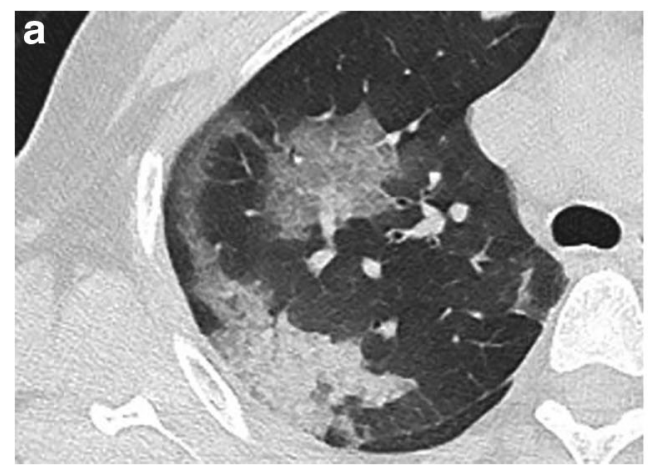

Fig. 4 A 40-year-old female with coronavirus disease 2019 in the progressive-stage group. a Axial non-contrast CT shows crazy-paving pattern in the anterior segment and consolidation in the posterior segment of right upper lobe. $\mathbf{b}$ Consolidations are noted in the bilateral lower lobes

were the main imaging finding $(68.4 \%)$ and were distributed in the periphery of the lung $(71.8 \%)$, with interlobular septal thickening $(24.2 \%)$ and intralobular interstitial hyperplasia $(32.2 \%)$, also called the crazy-paving pattern. These findings are very similar to the CT manifestations of COVID-19 [20]. For pneumonia caused by MERS-CoV, GGO was also found on the chest $\mathrm{CT}$ images and was mainly distributed in the subpleural and basilar lung regions [21, 22]. Interestingly, pleural effusion was rare and no tree-in-bud pattern or cavitation was found in pneumonia patients infected by the three kinds of coronaviruses. In addition, we found that only one case of COVID-19 (1.6\%) presented as consolidation without GGO. Compared with the large amount of consolidation that appears in the early stage of SARS, we speculate that the pathogenicity of SARS-CoV-2 may be less than that of SARS-CoV [23]. Thus, it is necessary to fully understand and discriminate the CT features of COVID-19 at an early stage, which is helpful for the timely isolation and respiratory care of patients and early implementation of infection prevention measures.

In addition to $\mathrm{GGO}$, another important $\mathrm{CT}$ characteristic of COVID-19 was multiple GGOs with consolidations, accompanied by interlobular septal thickening, mainly in the

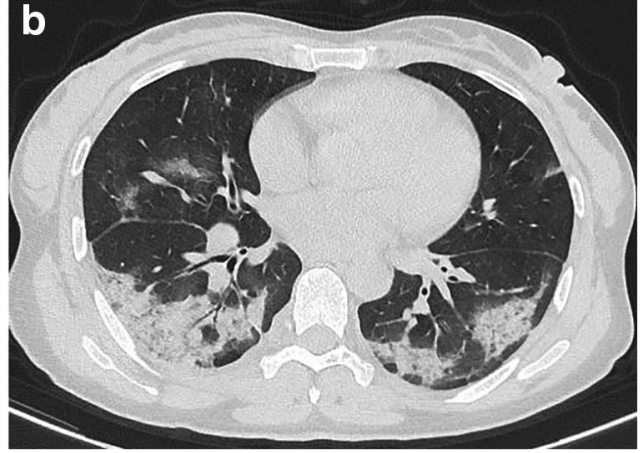

mainly in the peripheral regions, accompanied with subpleural curvilinear line. $\mathrm{CT}$ score $=3$ (right upper lobe $)+1$ (right middle lobe $)+3$ (right lower lobe $)+1$ (left upper lobe $)+3($ left lower lobe $)=11$

peripheral part of the lung. These basic CT features were similar to the findings reported by Huang et al, whose study showed that multiple GGOs with partial consolidation appeared bilaterally on the chest CT images of COVID-19 patients [7]. Similarly, in another chest CT study of a 33-year-old patient diagnosed with COVID-19 in Wuhan, China, multiple GGOs distributed in both the peripheral lungs were found [5]. Therefore, we suggest that multiple GGOs with consolidations mainly in the periphery of the lung are a basic CT feature of COVID-19, which is helpful for the initial screening of COVID-19.

Unexpectedly, the halo sign (20.6\%) was the only significantly different CT manifestation in the early stage of COVID-19 among all the CT findings. In our study, the halo sign appeared in only the early stage of COVID-19. We hypothesized that the halo sign rapidly changed into GGO within 1 week due to the aggravation of COVID-19, and no other new halo sign had been formed in the short term. In addition, there were no significant differences in the other CT findings or distribution of lesions between the two stages. For these findings, we speculated that the speed of GGO formation was due to the damage to the alveolar epithelium caused by the virus and may be similar to that of consolidation caused by an

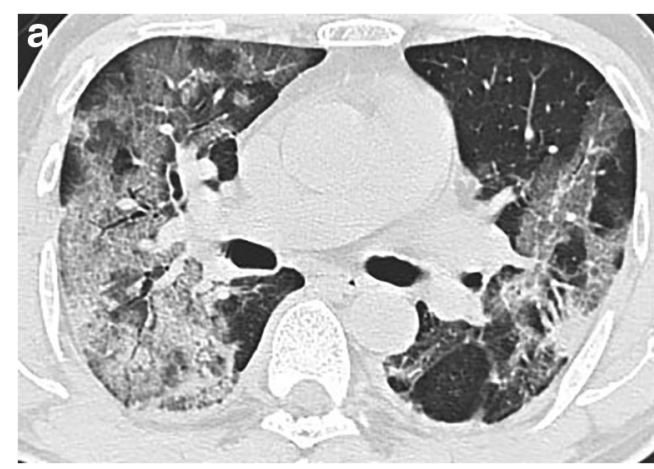

Fig. 5 A 60-year-old male with coronavirus disease 2019 in the progressive-stage group. a Axial non-contrast CT in pulmonary window shows diffuse ground-glass opacities with consolidations in bilateral lungs. b Axial non-contrast CT in mediastinal window shows

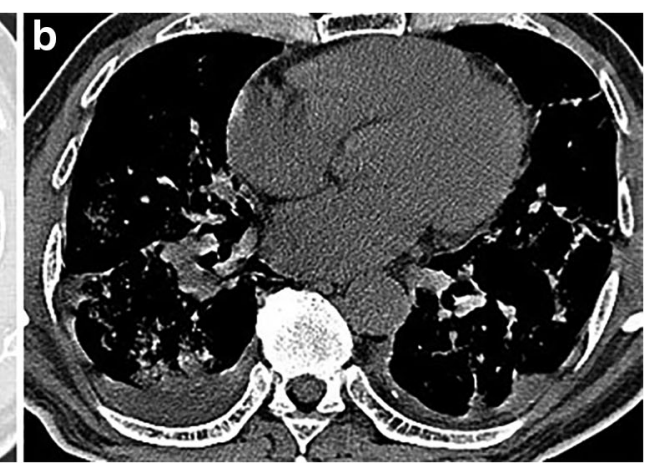

consolidations in bilateral lungs, with bilateral pleural effusion. CT score $=5$ (right upper lobe $)+4$ (right middle lobe $)+4$ (right lower lobe $)+3($ left upper lobe $)+2($ left lower lobe $)=18$ 
Fig. 6 The CT score of each lobe in the early-stage group and progressive-stage group
250

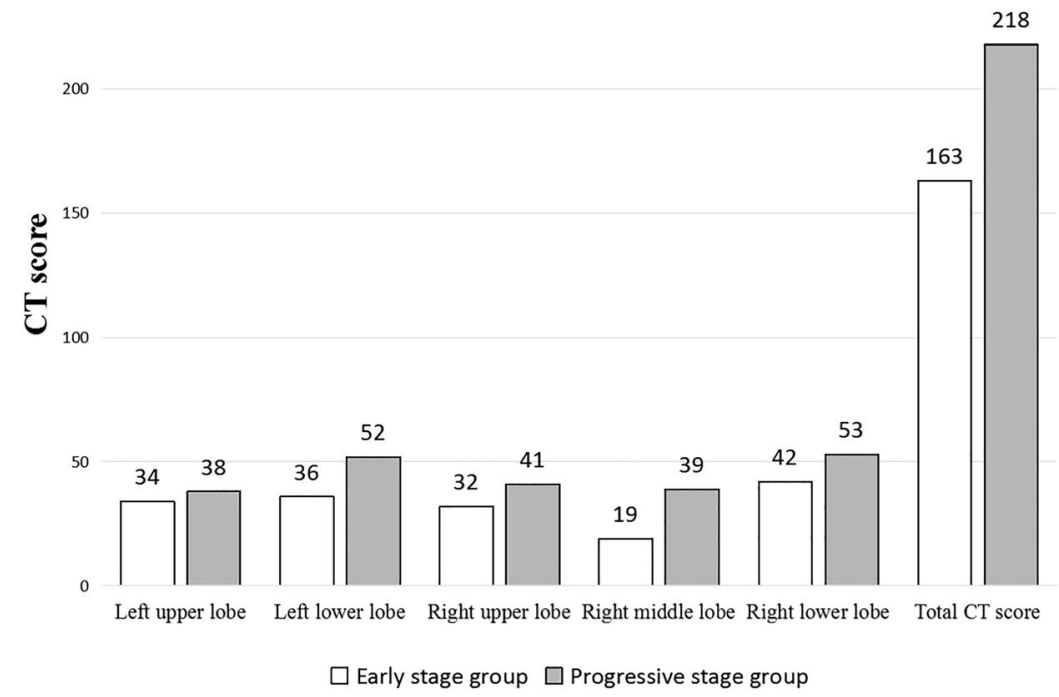

inflammatory reaction of the lung tissue, resulting in a change in different types of lesions during the synchronous increase in opacification. In pneumonia caused by SARS-CoV, Ooi et al found that GGO and consolidation formed in the first week of SARS-CoV infection increased in the second week, and only residual strips and reticular shadows were seen in the fourth week [15]. Therefore, the CT manifestations of COVID-19 are different at different stages, which helps the differential diagnosis of COVID-19.

Furthermore, our results showed that the $\mathrm{CT}$ scores in the progressive-stage group were significantly greater than those in the early-stage group. Further analysis showed that the CT scores were positively correlated with the maximum diameters of the lesions $(r=0.531)$, indicating that the range of lesions in the progressive stage was larger than and the degree of inflammation was more serious than that in the early stage. These results also showed that without any antiviral treatment, COVID-19 continued to progress 5-7 days after the onset of symptoms, without any improvement trend. Therefore, necessary clinical measures, including antiviral and supportive therapies, should be administered to prevent

Table 3 Correlation of clinical variables with CT score in patients with COVID-19

\begin{tabular}{lc}
\hline Variables & CT score \\
\hline Age (years) & \\
$p$ value & 0.045 \\
$r$ value & 0.255 \\
Max diameter of lesion $(\mathrm{cm})$ & \\
$p$ value & $<0.001$ \\
$r$ value & 0.531 \\
\hline
\end{tabular}

COVID-19, coronavirus disease 2019 disease deterioration. Because of the lack of additional data, the correlation between the CT score and clinical classification and the prognosis of COVID-19 needs further study. Moreover, CT scores showed a significant positive correlation with ages in all 62 patients $(r=0.255)$. In a previous study on SARS, Grinblat et al considered that age was a risk factor for severe lung injury and poor prognosis of SARS [24]. Therefore, elderly patients need to be treated earlier and more actively than younger patients.

Our study had several limitations. First, because COVID19 is a novel disease and lacks a series and long-term CT data, we can analyze only the existing information in a retrospective analysis. Moreover, the time of chest $\mathrm{CT}$ examination after symptom onset was inconsistent, so it was difficult to summarize the CT appearances that could reflect the whole course of the disease. Second, the sample size in our study was relatively small, and there may be deviation in the comparison of the results of CT findings. Third, most of our cases were imported cases and some were secondary cases. Further research is needed to observe whether virus variation exists in the process of infection and whether virus variation will produce different in CT manifestations. Finally, there was no histopathological evidence from deceased patients.

In conclusion, our study showed that chest $\mathrm{CT}$ examination was very effective in detecting pulmonary parenchymal abnormalities in the natural course of COVID-19. Multiple GGOs with consolidations in the peripheral lung was the primary CT characteristic of COVID-19. The halo sign may be a special CT feature in the early stage of COVID-19, while a significantly increased CT score may indicate the aggravation of COVID-19 in the progressive stage. In patients with dyspnea and respiratory distress, $\mathrm{CT}$ examination is very effective in the preclinical screening of patients with COVID-19. 
Funding information This study was supported by a grant from the Natural Science Foundation of China (81401382).

\section{Compliance with ethical standards}

Guarantor The scientific guarantor of this publication is Wenbing Zeng.

Conflict of interest The authors of this manuscript declare no relationships with any companies whose products or services may be related to the subject matter of the article.

Statistics and biometry No complex statistical methods were necessary for this paper.

Informed consent Written informed consent was waived by the Institutional Review Board.

Ethical approval Institutional Review Board approval was obtained.

\section{Methodology}

- Retrospective

- Observational

- Multicenter study

\section{References}

1. Holshue ML, DeBolt C, Lindquist S et al (2020) First case of 2019 novel coronavirus in the United States. N Engl J Med 382(10):929-936

2. World Health Organization (2020) Statement on novel coronavirus in Thailand. World Health Organization, Geneva Available via https://www.who.int/news-room/detail/13-01-2020-whostatement-on-novel-coronavirus-in-thailand. Accessed 12 Jan 2020

3. World Health Organization (2020) WHO confirms first cases of novel coronavirus (2019-nCoV) in the Eastern Mediterranean Region. World Health Organization, Geneva Available via http:// www.emro.who.int/media/news/who-confirms-first-cases-ofnovel-coronavirus-2019-ncov-in-the-eastern-mediterraneanregion.html. Accessed 29 Jan 2020

4. World Health Organization (2020) Readiness is the key to detect, combat spread of the new coronavirus. World Health Organization, Geneva Available via https://www.who.int/southeastasia/news/ detail/27-01-2020-readiness-is-the-key-to-detect-combat-spreadof-the-new-coronavirus. Accessed 27 Jan 2020

5. Lei J, Li J, Li X, Qi X (2020) CT imaging of the 2019 novel coronavirus (2019-nCoV) pneumonia. Radiology. https://doi.org/ 10.1148/radiol.2020200236:200236

6. Chen N, Zhou M, Dong X et al (2020) Epidemiological and clinical characteristics of 99 cases of 2019 novel coronavirus pneumonia in Wuhan, China: a descriptive study. Lancet 395(10223):507-513

7. Huang C, Wang Y, Li X et al (2020) Clinical features of patients infected with 2019 novel coronavirus in Wuhan, China. Lancet 395(10223):497-506

8. Yin Y, Wunderink RG (2018) MERS, SARS and other coronaviruses as causes of pneumonia. Respirology 23:130-137

9. World Health Organization (2020) Global surveillance for human infection with coronavirus disease (COVID-19). World Health Organization, Geneva Available via https:/www.who.int/ publications-detail/global-surveillance-for-human-infection-withnovel-coronavirus-(2019-ncov). Accessed 27 Feb 2020

10. Chan JF, Yuan S, Kok KH et al (2020) A familial cluster of pneumonia associated with the 2019 novel coronavirus indicating person-to-person transmission: a study of a family cluster. Lancet 395(10223):514-523

11. Li Q, Guan X, Wu P et al (2020) Early transmission dynamics in Wuhan, China, of novel coronavirus-infected pneumonia. N Engl J Med. https://doi.org/10.1056/NEJMoa2001316

12. People's Medical Publishing House; Committee of the Infectious Diseases Radiology Group of Chinese Society of Radiology; Committee of the Infectious Diseases Radiology Section of Chinese Medical Doctor Association; Infectious Disease Imaging Group, Infectious Disease Branch, Chinese Research Hospital Association; Imaging Committee of Chinese Association for the Prevention and Treatment of STD AIDS Infection (Infectious Disease); Infectious Diseases Group, General Radiological Equipment Committee, China Association of Medical Equipment; Beijing Imaging Diagnosis and Treatment Technology Innovation Alliance, Li HJ, Xu HB (2020) Guideline for imaging diagnosis of novel coronavirus $(2019-\mathrm{nCoV})$ infected pneumonia (1st edition 2020). New Medicine 30:22-34

13. Wormanns D, Hamer OW (2015) Glossary of terms for thoracic imaging-German version of the Fleischner Society recommendations. Rofo 187(8):638-661

14. Hansell DM, Bankier AA, MacMahon H, McLoud TC, Müller NL, Remy J (2008) Fleischner Society: glossary of terms for thoracic imaging. Radiology 246:697-722

15. Ooi GC, Khong PL, Müller NL et al (2004) Severe acute respiratory syndrome: temporal lung changes at thin-section CT in 30 patients. Radiology 230:836-844

16. Chang YC, Yu CJ, Chang SC et al (2005) Pulmonary sequelae in convalescent patients after severe acute respiratory syndrome: evaluation with thin-section CT. Radiology 236:1067-1075

17. Zhou P, Yang XL, Wang XG et al (2020) A pneumonia outbreak associated with a new coronavirus of probable bat origin. Nature. https://doi.org/10.1038/s41586-020-2012-7

18. Lu R, Zhao X, Li J et al (2020) Genomic characterisation and epidemiology of 2019 novel coronavirus: implications for virus origins and receptor binding. Lancet 395(10224):565-574

19. Koo HJ, Lim S, Choe J, Choi SH, Sung H, Do KH (2018) Radiographic and CT features of viral pneumonia. Radiographics 38:719-739

20. Wong KT, Antonio GE, Hui DS et al (2003) Thin-section CT of severe acute respiratory syndrome: evaluation of 73 patients exposed to or with the disease. Radiology 228:395-400

21. Das KM, Lee EY, Enani MA et al (2015) CT correlation with outcomes in 15 patients with acute Middle East respiratory syndrome coronavirus. AJR Am J Roentgenol 204:736-742

22. Ajlan AM, Ahyad RA, Jamjoom LG, Alharthy A, Madani TA (2014) Middle East respiratory syndrome coronavirus (MERS$\mathrm{CoV}$ ) infection: chest CT findings. AJR Am J Roentgenol 203: 782-787

23. Munster VJ, Koopmans M, van Doremalen N, van Riel D, de Wit E (2020) A novel coronavirus emerging in China - key questions for impact assessment. N Engl J Med 382(8):692-694

24. Grinblat L, Shulman H, Glickman A, Matukas L, Paul N (2003) Severe acute respiratory syndrome: radiographic review of 40 probable cases in Toronto, Canada. Radiology 228:802-809

Publisher's note Springer Nature remains neutral with regard to jurisdictional claims in published maps and institutional affiliations. 